\title{
A Study of Systems with Multiple Operating Levels, Probabilistic Thresholds and Hysteresis
}

\author{
Alexandre Brandwajn,Thomas Begin,Hind Castel-Taleb and Tulin Atmaca
}

\begin{abstract}
Current architecture of many computer systems relies on dynamic allocation of a pool of resources according to workload conditions to meet specific performance objectives while minimizing cost (e.g., energy or billing). In such systems, different levels of operation may be defined, and switching between operating levels occurs at certain thresholds of system congestion. To avoid rapid oscillations between levels of service, "hysteresis" is introduced by using different thresholds for increasing and decreasing workload levels, respectively.

We propose a model of such systems with general arrivals, arbitrary number of servers and operating levels where each higher operating level may correspond to an arbitrary number of additional servers and soft (i.e. non-deterministic) thresholds to account for "inertia" in switching between operating levels. In our model, request service times are assumed to be memoryless and server processing rates may be a function of the current operating level and of the number of requests (users) in the system. Additionally, we allow for delays in the activation of additional operating levels. We use simple mathematics to obtain a semi-numerical solution of our model. We illustrate the versatility of our model using several case study examples inspired by features of real systems. In particular, we explore optimal thresholds as a tradeoff between performance and energy consumption.
\end{abstract}

Index Terms-Multi-server systems, multiple operating levels, hysteresis, probabilistic thresholds, general arrivals, activation delays.

\section{INTRODUCTION}

C URRENT architecture of computer systems and services tends to rely on dynamic allocation of a pool of resources (such as Virtual Machines, processors, storage, etc.) under varying workload conditions in order to meet specific performance objectives while at the same time minimizing cost (e.g., in terms of energy or billing). Examples range from cloud computing [1] and virtualization environments such as VMware [2] to enterprise Operating Systems such as IBMs AIX [3] or Virtual Network Switches in the context of future generation Network Function Virtualization (NFV) [4].

To adapt the number of discrete resources to dynamically varying workloads, different levels of operation are defined and switching between operating levels occurs at certain thresholds. These thresholds may correspond to resource utilization or some other measure of system congestion. To avoid overreacting to spurious workload changes, some "inertia" is introduced through the use of averaging such as sliding window or exponential smoothing, and to avoid rapid oscillations between levels of service, "hysteresis" is introduced by defining different thresholds for increasing and decreasing workload levels respectively.

To our knowledge, the bulk of theoretical analysis of systems with multiple operating levels in the literature is limited to the case where each increase (respectively, decrease) in operating level corresponds to adding (respec-

- A. Brandwajn is Professor Emeritus at Baskin School of Engineering, University of California Santa Cruz, USA and he is employed by Pallas International Corporation, San Jose, USA.

- T. Begin is with Université Lyon 1 / LIP (UMR Inria, ENS Lyon CNRS, $U(B)$, France.

- H. Castel-Taleb and T. Atmaca are with SAMOVAR, Télécom SudParis, CNRS, Université Paris-Saclay, 9 rue Charles Fourier, Évry, France.

Manuscript received Month Day, Year; revised Month Day, Year. tively, removing) a single server, there is no inertia in thresholds (deterministic instantaneous thresholds), and request arrivals come from a Poisson source (single or bulk).

Ibe and Keilson [5] derived a closed-form solution for the steady-state distribution of the number of requests in the system through the use of Greens function under the assumption of Poisson arrivals and hard deterministic thresholds where additional servers are allocated immediately as specific values of the number of requests are exceeded. Their numerical results are limited to 3 servers. In 1997, Golubchik and Lui | $|6|$ used a combination of the stochastic complementation and matrix geometric methods to derive upper and lower bounds on the performance of such systems with hard deterministic thresholds, Poisson arrivals, and single server allocation per operating level change. Their work accounts for a possible delay in the activation of each operating level. The numerical results in their paper are limited to 5 servers. A couple of years later, the same authors [7] used the stochastic complementation method to obtain an exact solution for homogenous and heterogeneous servers with single and bulk Poisson arrivals. Numerical results in this paper are again limited to 5 servers. In 2000, Le Ny and Tuffin [8] proposed an exact solution for the case of heterogeneous servers with Poisson arrivals. Their cuts method uses simpler mathematics than previous work in the literature to obtain the steady-state distribution of the number of requests in the system. No numerical examples are presented in their paper.

More recently, Mitrani [9], [10] considered a model of a system with Poisson request arrivals and two blocks of servers where the reserve block is activated and deactivated according to forward and backward thresholds. The paper focuses on the selection of thresholds and the number of servers in the reserve block so as to optimize 
a cost function integrating system performance and energy consumption. Ait-Salaht and Castel-Taleb [11], [12] consider a model of a node in a cloud system with hysteresis where virtual machines are added or removed one at a time. They use stochastic bounding to derive approximate steady-state probabilities of the number of requests in such a node with Poisson arrivals single and bulk.

Our contribution is to propose a model of systems with hysteresis, general arrivals, arbitrary number of servers and operating levels where as the operating levels increase the number of servers may increase in arbitrary increments and soft (i.e., non-deterministic) thresholds to account for "inertia" in switching between operating levels. We assume memoryless request service times, however, unlike in previous work, to account for potential speed degradation as the number of servers increases, in our model request completion rates with multiple servers are not necessarily multiples of single server rates. We also allow for delays in the activation of additional operating levels. We use simple mathematics to obtain a semi-numerical solution of our model.

In the next section we describe in more detail the model considered and we outline the proposed solution approach. Section 3 is devoted to several case studies, which illustrate possible applications of our model. Section 4 concludes this paper.

\section{Model AND SOLUTION OUTLINE}

\subsection{Model description}

We consider a system with two possible types of request arrival processes (see Figure 1). In the first type, the times between consecutive requests are assumed to be memoryless [13] with a state-dependent rate $\lambda(n)$ when the current number of requests in the system is $n$. In addition to including the standard Poisson arrivals, such a quasi-Poisson arrival process can be a good representation of arrivals generated by a set of discrete request sources. In the second type of arrivals considered, times between consecutive arrivals are assumed to be independent but can have a general distribution. Specifically, times between request arrivals are distributed according to a phase-type distribution [14] with $a$ exponential phases. We denote by $\tau_{j}$ the probability that the arrival process starts in phase $j(j=1, \ldots, a)$, by $\lambda_{j}$ the intensity of phase $j$ and by $\hat{r}_{j}$ the probability that the arrival process completes after phase $j . r_{o j}$ denotes the probability that the arrival process continues to phase $j$ upon completion of phase $o$. Such phase-type distributions can represent arbitrarily closely any distribution [15] and readily available methods exist to map theoretical or empirical distributions onto them [16], [17].

As shown in Figure 1, the system has a maximum of $C$ homogeneous servers and can accommodate a maximum of $N$ requests (system capacity). Although the capacity of any practical system is finite, we treat also the case where there is no limitation on the number of requests. The system operates at $L$ different levels, each level $\ell=1, \ldots, L$ corresponds to a given number of active servers $c_{\ell}$ with $c_{L}=C$ (increasing operating levels correspond to increasing numbers of servers). We assume that request service times have a memoryless distribution and we denote by $\mu(n, \ell)$ the rate of request completions when the current number of requests is $n$ and the current operating level is $\ell$. This allows, for example, in models of multiprocessor systems to account for the fact that the service rate of each processor may degrade as the number of active processors increases due to interprocessor interference in multiprocessor environments. We assume that the activation of additional servers corresponding to the next system operating level is not necessarily instantaneous. We denote by $1 / \omega$ the mean time to activate a new operating level and we assume that the level activation time is exponentially distributed. Thus, at any time the system could be operating at level $\ell$ and there could be $k=0, \ldots, L-\ell$ levels whose activation is pending, i.e. whose activation has been scheduled but has not yet become effective due to activation delays. Note that if the activation of operating levels is instantaneous, there are never any pending levels and we must have $k=0$.. For our purposes, we assume that level deactivation is instantaneous.

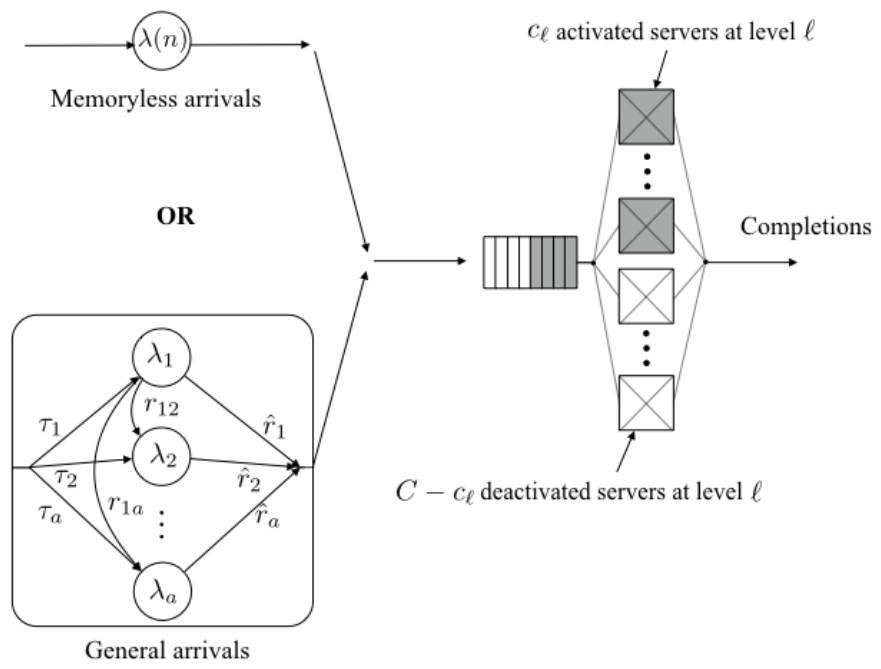

Fig. 1: System with a total of $C$ servers and $L$ operating levels.

The current state of our system (but not that of the arrival process in the case of general arrivals) is defined by the triplet $(n, \ell, k)$ where $n=0, \ldots, N$ is the number of requests in the system, $\ell=1, \ldots, L$ is the current operating level and $k=0, \ldots, L-\ell$ is the number of levels whose activation is pending. With each state $(n, \ell, k)$ (except those for which $\ell+k=L$ or $n=N$ ) we associate a probability denoted by $\gamma(n, \ell, k)$ that the activation of an additional operating level will be requested if the current number of requests increases from $n$ to $n+1$. Similarly, with each state except those for which $\ell=1$ or $n=0$ we associate a probability denoted by $\delta(n, \ell, k)$ that the deactivation of an operating level will happen if the current number of requests decreases from $n$ to $n-1$. Thus, depending on the values of these probabilities, an increase (respectively, decrease) in the current number of users can trigger a request for an increase (respectively, decrease) in the system operating level. Note that we assume that deactivation requests are applied to pending levels (if any) first, i.e. if the activation of an operating level is still pending due to activation delays when a deactivation request is triggered, 
the activation of the pending level is simply cancelled. If the values of these probabilities are simply 0 or 1 , the above description defaults to classical "hard" forward and backward thresholds. The use of other values corresponds to "soft" thresholds allowing one to model "inertia" as well as some variability in triggering the activation and deactivation of operating levels. In this paper we use the terms "soft" thresholds and probabilistic or non-deterministic thresholds interchangeably. Note in passing that such "soft" thresholds are quite common beyond computer applications, e.g., in supermarket policies for adding checkout clerks based on the length of the queue. The performance metrics of interest in our model include the mean response time for a request, attained request throughput, as well as the mean number of active servers, and the fraction of time the system operates at each level. With a finite system capacity, the loss probability may also be of interest. Principal notation used in this paper is summarized in Table 1.

\subsection{Outline of model solution with memoryless arrivals}

We start by considering the case of memoryless arrivals with rate $\lambda(n)$ for which the triplet $(n, \ell, k)$ is sufficient to describe the state of our system. We let $p(n, \ell, k)$ be the steady-state probability of the system being in state $(n, \ell, k)$. It is easy to derive the corresponding balance equations. For $n>1$, we have

$$
\begin{array}{r}
p(n, \ell, k)[\lambda(n)+\mu(n, \ell)+k w]= \\
p(n-1, \ell, k) \lambda(n-1)[1-\gamma(n-1, \ell, k)] \\
+p(n-1, \ell, k-1) \lambda(n-1) \gamma(n-1, \ell, k-1) \\
+p(n, \ell-1, k+1)(k+1) \omega \\
+p(n+1, \ell, k) \mu(n+1, \ell)[1-\delta(n+1, \ell, k)] \\
+p(n+1, \ell, k+1) \mu(n+1, \ell) \delta(n+1, \ell, k+1) \\
+p(n+1, \ell, k) \mu(n+1, \ell+1) \delta(n+1, \ell+1, k)
\end{array}
$$

For $n=1$, we have

$$
\begin{array}{r}
p(1, \ell, k)[\lambda(1)+\mu(1, \ell)+k w]= \\
p(0, \ell, k) \lambda(0) \\
+p(1, \ell-1, k+1)(k+1) \omega \\
+p(2, \ell, k) \mu(2, \ell)[1-\delta(2, \ell, k)] \\
+p(2, \ell, k+1) \mu(2, \ell) \delta(2, \ell, k+1) \\
+p(2, \ell, k) \mu(2, \ell+1) \delta(2, \ell+1, k)
\end{array}
$$

In the above equations, impossible terms are assumed to vanish and the last term is present only for $k=0$. For $n=0$, we assume that the system operates at level 1 and that there are no pending level activations so that we have

\begin{tabular}{|c|c|}
\hline \multicolumn{2}{|r|}{ Main notation } \\
\hline C & Number of servers \\
\hline$N$ & System capacity \\
\hline$L$ & Number of operating levels \\
\hline$n$ & Current number of requests in the system \\
\hline$\ell$ & Current operating level \\
\hline$c_{\ell}$ & Number of active servers at level $\ell$ \\
\hline$\mu(n, \ell)$ & Rate of request completions given $n$ and $\ell$ \\
\hline$\lambda(n)$ & $\begin{array}{l}\text { Rate of request arrivals given } n \text { (case of memoryless } \\
\text { arrivals) }\end{array}$ \\
\hline $1 / \omega$ & Mean time to activate a new operating level \\
\hline$k$ & Number of operating levels whose activation is pending \\
\hline$\gamma(n, \ell, k)$ & $\begin{array}{l}\text { Probability of activating an additional operating level if } \\
n \text { increases to } n+1\end{array}$ \\
\hline$\delta(n, \ell, k)$ & $\begin{array}{l}\text { Probability of deactivating an additional operating level } \\
\text { if } n \text { decreases to } n-1\end{array}$ \\
\hline \multicolumn{2}{|r|}{ Intermediate quantities in the solution } \\
\hline$p(n, \ell, k)$ & $\begin{array}{l}\text { Steady-state probability of the system being in state } \\
(n, \ell, k)\end{array}$ \\
\hline$p(n)$ & $\begin{array}{l}\text { Marginal steady-state probability of having } n \text { requests } \\
\text { in the system }\end{array}$ \\
\hline$p(\ell, k \mid n)$ & $\begin{array}{l}\text { Conditional probability of being at operating level } \ell \\
\text { with } k \text { pending levels given } n\end{array}$ \\
\hline$u(n)$ & Conditional rate of request completion given $n$ \\
\hline \multicolumn{2}{|r|}{ Performance metrics } \\
\hline $\bar{n}$ & Mean number of requests in the system \\
\hline$\theta$ & Attained request throughput \\
\hline$R$ & Mean request response time \\
\hline$p(\ell)$ & Fraction of time the system spends on operating level $\ell$ \\
\hline $\bar{c}$ & Mean number of busy servers \\
\hline$P_{A}(n)$ & $\begin{array}{l}\text { Probability that a request finds the system with } n \text { re- } \\
\text { quests upon arrival }\end{array}$ \\
\hline \multicolumn{2}{|c|}{ Additional quantities for general arrivals (phase-type distribution) } \\
\hline$a$ & Number of exponential phases in the arrival process \\
\hline$j$ & Current phase of the arrival process \\
\hline$\tau_{j}$ & Probability that the arrival process starts in phase $j$ \\
\hline$\lambda_{j}$ & Intensity of phase $j$ \\
\hline$\hat{r}_{j}$ & $\begin{array}{l}\text { Probability that the arrival process completes after } \\
\text { phase } j\end{array}$ \\
\hline$r_{o j}$ & $\begin{array}{l}\text { Probability that the arrival process continues to phase } j \\
\text { upon completion of phase } o\end{array}$ \\
\hline \multicolumn{2}{|c|}{$\begin{array}{c}p(n, \ell, k, j) \text { Steady-state probability of the system being in state } \\
(n, \ell, k) \text { and the arrival process in phase } j\end{array}$} \\
\hline$\alpha(n)$ & $\begin{array}{l}\text { Conditional rate of arrivals given that there are } n \text { re- } \\
\text { quests in system }\end{array}$ \\
\hline \multicolumn{2}{|r|}{ Case studies } \\
\hline$K$ & $\begin{array}{l}\text { Number of exponential request sources (case of memo- } \\
\text { ryless arrivals) }\end{array}$ \\
\hline$\phi$ & $\begin{array}{l}\text { Unitary rate of an active source (case of memoryless } \\
\text { arrivals) }\end{array}$ \\
\hline$T$ & Mean request execution time \\
\hline
\end{tabular}
$p(0,1,0)=\sum_{\ell=1}^{L} \sum_{k=0}^{L-\ell} p(1, \ell, k) \mu(1, \ell)$.

Denote by $p(n)$ the marginal steady-state probability that there are $n$ requests in the system and by $p(\ell, k \mid n)$ the conditional probability that the current operating level is $\ell$ and there are $k$ levels pending given $n$. We then have

$$
p(n, \ell, k)=p(\ell, k \mid n) p(n)
$$

The conditional rate of request completions given $n$, denoted by $u(n)$, can be written as

$$
u(n)=\sum_{\ell=1}^{L} \mu(n, \ell)\left(\sum_{k=0}^{L-\ell} p(\ell, k \mid n)\right)
$$

TABLE 1: Notation used.

and the probability $p(n)$ can be expressed as

$$
p(n)=\frac{1}{G} \prod_{i=1}^{n} \frac{\lambda(i-1)}{u(i)}, \quad n=0,1, \ldots
$$

In (3) empty products are assumed to be equal to 1 and $G$ is a normalizing constant such that $\sum_{n=0}^{N} p(n)=1$. Note that equation (3) can be shown to be exact by using (1) in the balance equations for $p(n, \ell, k)$ and simply summing over all values of $\ell$ and $k$ for each $n$ (cf. [18]). Using equations (1) and (3) in the balance equations for $p(n, \ell, k)$, we readily obtain a set of equations for the con- 
ditional probabilities $p(\ell, k \mid n)$. We then use simple fixedpoint iteration to solve this set of equations numerically. We refer the interested reader to Section 2.4 for more detail about the proposed solution approach and its advantages. Having obtained the $p(\ell, k \mid n)$ and hence the equivalent completion rates $u(n)$ from (2), we compute the steadystate probabilities $p(n)$ using (3). From here, it is a straightforward matter to compute the mean number of requests in the system as $\bar{n}=\sum_{n=1}^{N} n p(n)$, the attained request throughput as $\theta=\sum_{n=1}^{N} p(n) u(n)$ and the mean request response time as $R=\bar{n} / \theta$. The fraction of time the system spends on each operating level can be computed as $p(\ell)=\sum_{n=0}^{N} p(n)\left(\sum_{k=0}^{L-\ell} p(\ell, k \mid n)\right)$ and the mean number of busy servers is given by $\bar{c}=\sum_{\ell=1}^{L} p(\ell) c_{\ell}$. The state probabilities seen by an arriving request are given by $P_{A}(n)=\frac{\lambda(n) p(n)}{\sum_{i=0}^{N} \lambda(i) p(i)}$ so that, when the system capacity is finite, the loss probability can be obtained as $P_{A}(N)$.

\subsection{Outline of model solution with general arrivals}

We now consider the case where the times between arrivals have a general distribution (phase-type, independent and identically distributed). With such phase-type arrivals, the state description needs to be extended to include the current phase of the arrival process, $j, j=1, \ldots, a$. We can then define the steady-state probability of the new full system state $p(n, \ell, k, j)$ where $n, \ell$ and $k$ have the same meaning as before. One can derive balance equations for $p(n, \ell, k, j)$, transform them into equations for the conditional probability $p(\ell, k, j \mid n)$ and solve the latter using fixed-point iteration in a way quite analogous to the one described before for memoryless arrivals.

As an alternative to this exact solution, we propose an even simpler "divide and conquer" approach, which has the added advantage of using previously developed solutions. We replace our model with state description $p(n, \ell, k, j)$ by two simpler models. In the first one, phase-type arrivals are replaced by state-dependent memoryless arrival rate $\alpha(n)$ while all other system aspects are fully represented. In effect, this model is the same as the one considered before for memoryless arrivals with state description $(n, \ell, k)$. In the second model, we use the state description $(n, j)$ to fully represent the phase-type times between arrivals but we replace the remainder of the system by the equivalent conditional rate of request completions $u(n)$ obtained from the solution of our first model. In effect, our second model is a simple $\mathrm{Ph} / \mathrm{M} / \mathrm{C}$ type of queue, which can be easily solved using a simple numerically stable recurrence [19]. The solution of this model produces the steady-state probability $p(n, j)$ for the state description $(n, j)$ and the equivalent statedependent rate of request arrivals $\alpha(n)=\frac{\sum_{j=1}^{a} \lambda_{j} \hat{r}_{j} p(n, j)}{\sum_{j=1}^{a} p(n, j)}$. Since we need the $\alpha(n)$ from our second model in order to compute the $u(n)$ from our first model, needed in our second model, we naturally end up with a fixed-point iteration between our two models as shown in Figure 2. We end our iteration when the mean numbers of requests in the system computed from both models are sufficiently close.

Algorithm 1 summarizes this fixed-point iteration between our two simpler models. $\overline{\text { Algorithm } 1 \text { Solution of model with general arrivals via }}$ iteration between two simpler models

1: Initialize the arrival rate values $\alpha(n)$ to the inverse of the mean time between arrivals for all $n=0, \ldots, N$.

2: Solve the model with state-dependent memoryless arrivals using the current values of $\alpha(n)$.

a: Obtain current values for $p(\ell, k \mid n)$ and $p(n)$, as well as the equivalent service rate $u(n)$.

b: Compute current value of $\bar{n}$ from this model.

3: Solve the $P h / M / C$ queue (our second model) using the current values of $u(n)$ rate from Step 2 as service rates.

a: Obtain current values for $p(n, j)$ and for $\alpha(n)$.

b: Compute the current value of $\bar{n}$ from this model $(\bar{n}=$ $\left.\sum_{n=1}^{N} n\left(\sum_{j=1}^{a} p(n, j)\right)\right)$.

4: If the values of $\bar{n}$ from Step 2 and Step 3 deviate by less than $\epsilon>0$ then stop the iteration, otherwise go to Step 2 .

5: Use the values of $p(\ell, k \mid n)$ and $p(n)$ from last execution of Step 2 as the solution of the model.

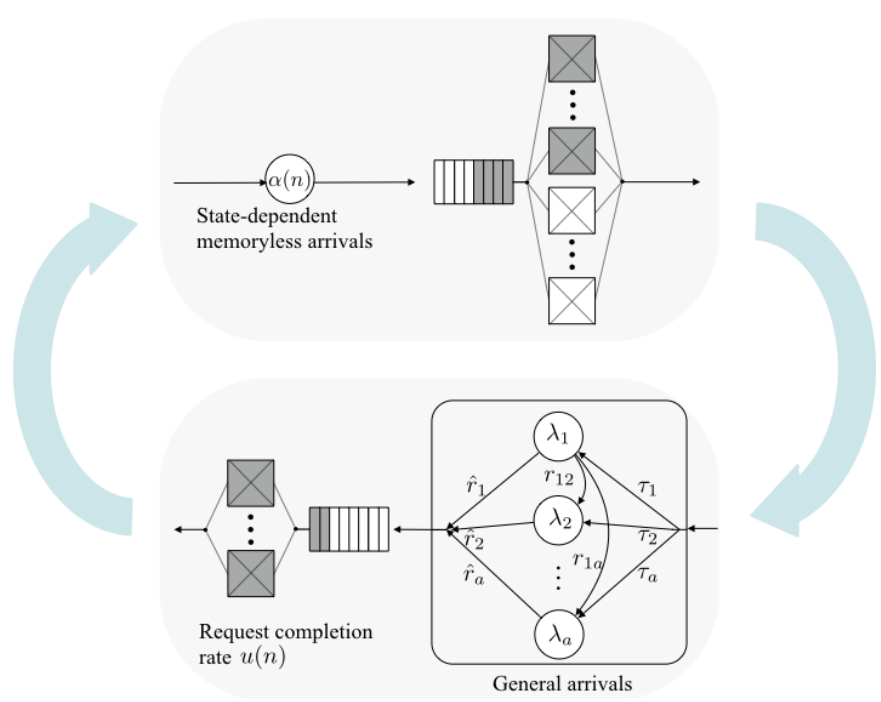

Fig. 2: Iteration between two simpler models in the case of general arrivals.

Here, the state probabilities viewed by an arriving request are given by $P_{A}(n)=\frac{\alpha(n) p(n)}{\sum_{i=0}^{N} \alpha(i) p(i)}$. Note that strictly speaking, for our first model to be exact, we would need a rate of arrivals $\alpha(n, \ell, k)$. We introduce an approximation by assuming that the equivalent conditional rate of arrivals depends only on the current number of requests in the system $n$ but not on the current system operating level or the number of pending levels. Similarly, strictly speaking we would need an equivalent service rate $u(n, j)$ for our second model to be exact. Again, we introduce an approximation by using a service rate that depends only on the current number of requests but not on the current phase of the arrival process. To summarize, the approximations introduced by our "divide and conquer" approach are $\alpha(n, \ell, k) \simeq \alpha(n)$ and $u(n, j) \simeq u(n)$. It has been our experience that the inaccuracy introduced by this type of approximation is generally quite small (cf. [20|) and the number of iterations between models required for convergence is typically below 10 . 


\subsection{Advantages and simple implementation of the pro- posed solution}

Transforming the balance equations for our model into equations for the conditional probabilities $p(\ell, k \mid n)$ we get for $n>1$

$$
\begin{array}{r}
p(\ell, k \mid n)[\lambda(n)+\mu(n, \ell)+k w]= \\
p(\ell, k \mid n-1) u(n)[1-\gamma(n-1, \ell, k)] \\
+p(\ell, k-1 \mid n-1) u(n) \gamma(n-1, \ell, k-1) \\
+p(\ell-1, k+1 \mid n)(k+1) \omega
\end{array}
$$$$
+p(\ell, k \mid n+1) \mu(n+1, \ell)[1-\delta(n+1, \ell, k)] \lambda(n) / u(n+1)
$$$$
+p(\ell, k+1 \mid n+1) \mu(n+1, \ell) \delta(n+1, \ell, k+1) \lambda(n) / u(n+1)
$$$$
+p(\ell, k \mid n+1) \mu(n+1, \ell+1) \delta(n+1, \ell+1, k) \lambda(n) / u(n+1)
$$

Similarly, for $n=1$ we get

$$
\begin{array}{r}
p(\ell, k \mid 1)[\lambda(1)+\mu(1, \ell)+k w]=u(1) \\
+p(\ell-1, k+1 \mid 1)(k+1) \omega \\
+p(\ell, k \mid 2) \mu(2, \ell)[1-\delta(2, \ell, k)] \lambda(1) / u(2) \\
+p(\ell, k+1 \mid 2) \mu(2, \ell) \delta(2, \ell, k+1) \lambda(1) / u(2) \\
+p(\ell, k \mid 2) \mu(2, \ell+1) \delta(2, \ell+1, k) \lambda(1) / u(2)
\end{array}
$$

As was the case for the balance equations, impossible terms are assumed to vanish and the last term in the above equations is present only for $k=0$. Note that $u(n)$ is given by formula (2) and we must have $\sum_{\ell=1}^{L} \sum_{k=0}^{L-\ell} p(\ell, k \mid n)=1$ for all values of $n$. These equations, considered in the order $n=1,2, \ldots$, can be solved using a simple fixed-point iteration. The use of conditional probabilities has the effect of partitioning the state space into independently normalized probabilities for each value of $n$. We believe that this has the potentially beneficial effect of enhancing the numerical stability of iterative solutions by reducing round-off errors [21]. In the case when there are no level activation delays, for any values of $n$ for which operating levels dont overlap, we have $p(\ell, 0 \mid n)=1$ for the single level $\ell$ corresponding to $n$. Thus, in models without activation delays, the use of conditional probabilities reduces the computational effort to sole regions where operating levels overlap. Note also that in the case of infinite population size $(N=\infty)$, the conditional probabilities $p(\ell, k \mid n)$ tend to a limiting distribution as $n \rightarrow \infty$. In practice, this asymptotic convergence tends to happen for reasonably small values of $n$ thus avoiding arbitrary truncation.

We now briefly outline a possible iterative solution of the conditional probability equations. Denote by the superscript the current iteration number in a fixed-point iteration. A simple implementation could start with a feasible set of initial values for the conditional probabilities $p^{0}(\ell, k \mid n)$ for $n=1,2, \ldots$ and the corresponding conditional rates of request completions $u^{0}(n)=\sum_{\ell=1}^{L} \mu(n, \ell) \sum_{k=0}^{L-\ell} p^{0}(\ell, k \mid n)$. Let $\pi(\ell, k \mid n)$ denote non-normalized values corresponding to $p^{i}(\ell, k \mid n)$. Then, enumerating system states in the order of increasing values of $\ell$ for consecutive increasing values of $n=1,2, \ldots$, at each iteration we can compute

$$
\begin{array}{r}
\pi^{i}(\ell, k \mid 1)=[\lambda(1)+\mu(1, \ell)+k w]^{-1}\left[u^{i-1}(1)\right. \\
+\pi^{i}(\ell-1, k+1 \mid 1)(k+1) \omega \\
+p^{i-1}(\ell, k \mid 2) \mu(2, \ell)[1-\delta(2, \ell, k)] \lambda(1) / u^{i-1}(2) \\
+p^{i-1}(\ell, k+1 \mid 2) \mu(2, \ell) \delta(2, \ell, k+1) \lambda(1) / u^{i-1}(2) \\
\left.+p^{i-1}(\ell, k \mid 2) \mu(2, \ell+1) \delta(2, \ell+1, k) \lambda(1) / u^{i-1}(2)\right]
\end{array}
$$

For $n>1$ we have

$$
\begin{array}{r}
\pi^{i}(\ell, k \mid n)=[\lambda(n)+\mu(n, \ell)+k w]^{-1} \\
{\left[p^{i}(\ell, k \mid n-1) u^{i-1}(n)[1-\gamma(n-1, \ell, k)]\right.} \\
+p^{i}(\ell, k-1 \mid n-1) u^{i-1}(n) \gamma(n-1, \ell, k-1) \\
+\pi^{i}(\ell-1, k+1 \mid n)(k+1) \omega \\
+p^{i-1}(\ell, k \mid n+1) \mu(n+1, \ell)[1-\delta(n+1, \ell, k)] \lambda(n) / u^{i-1}(n+1) \\
+p^{i-1}(\ell, k+1 \mid n+1) \mu(n+1, \ell) \delta(n+1, \ell, k+1) \lambda(n) / u^{i-1}(n+1) \\
\left.+p^{i-1}(\ell, k \mid n+1) \mu(n+1, \ell+1) \delta(n+1, \ell+1, k) \lambda(n) / u^{i-1}(n+1)\right]
\end{array}
$$

As before, impossible terms are assumed to vanish and the last term in the above equations is present only for $k=0$. In this approach, we immediately use newly computed values $\pi^{i}(\ell, k \mid n)$, then, as soon as all $\pi^{i}(\ell, k \mid n)$ values for a given $n=1,2, \ldots$ have been obtained, we normalize them to get $p^{i}(\ell, k \mid n)=\pi^{i}(\ell, k \mid n) / \sum_{\ell=1}^{L} \sum_{k=0}^{L-\ell} \pi^{i}(\ell, k \mid n)$. Of course, more sophisticated iterative schemes can easily be devised.

In the next section, we illustrate the versatility of our model using several examples derived from existing computer and network systems.

\section{Case studies}

\subsection{Example I: AIX SMT-like system}

In our first example we consider a system inspired by the Simultaneous Multithreading feature in IBMs AIX Operating System [22]. We assume that the system can operate at 3 levels where the corresponding numbers of logical processors are 2, 4 and 8 . We take the activation delay for levels to be negligible $(1 / \omega \simeq 0)$. To represent possible processor interference, we assume that the service rate of each processor degrades as the operating level increases. Specifically, we assume that the service rate of each processor degrades by a factor of 0.95 for each consecutive higher operating level, i.e. as the number of processors doubles. The base mean service time with a single processor is taken to be 1 . Our system functions with "soft" thresholds chosen so as to switch to the next higher operating level when the relative request response time is around 10 and switch back to the preceding level when the relative response time is around 7. We define the mean relative response time as the ratio of the mean request response time to the mean service time with a single active processor. Arrivals are assumed to come from a set of $K=256$ exponential request sources yielding a state-dependent arrival rate given by $\lambda(n)=(K-n) \phi$ where $\phi$ is the unitary request rate of an active source. The system capacity $N$ is taken to be greater or equal to the number of request sources so that there are no lost requests. For this example, we use directly the solution described for memoryless arrivals in Section 2.2.

Figure 3 shows the mean relative response time and the mean number of active processors for this example as a function of the unitary request rate of an active source $\phi$. We observe that the response time exhibits a characteristic "dip" and inflexion points as the system switches between its operating levels, while the mean number of processors increases with system load and then reaches its maximum value.

For all examples, only non-zero threshold values are specified. We used the following "soft" threshold values 


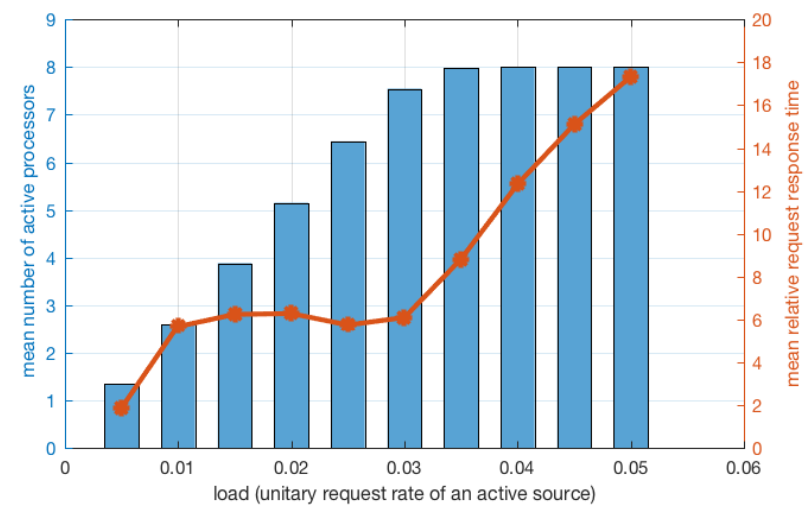

Fig. 3: Example I: mean relative request response time (right $\mathrm{y}$-axis) and mean number of active processors (left y-axis) as a function of load.

in this example: $\gamma(17,1,0)=1 / 3, \gamma(18,1,0)=2 / 3$, $\gamma(19,1,0)=1, \gamma(35,2,0)=1 / 3, \gamma(36,2,0)=2 / 3$, $\gamma(37,2,0)=1, \delta(14,2,0)=1 / 3, \delta(13,2,0)=2 / 3$, $\delta(12,2,0)=1, \delta(26,3,0)=1 / 3, \delta(25,3,0)=2 / 3$, $\delta(24,3,0)=1$. These threshold values were chosen so as to reproduce the type of response time behavior observed in certain actual AIX measurements.

\subsection{Example II: Virtual Switch-like system}

In our second example, we consider a system inspired by Virtual Switching systems (vSwitches) in Network Function Virtualization environments [4]. A class of such vSwitches |23| can dynamically enable processor cores to respond to varying packet workloads. Here, we assume that there are $C=8$ processors and $L=8$ operating levels with $c_{\ell}=\ell$, $\ell=1, \ldots, L$ i.e. a single processor core is added (respectively, removed) when switching to the next higher (respectively, lower) level. In this example, we use "hard" forward and backward thresholds with negligible level activation delay. Since it is well known that packet arrivals processes in computer networks tend to deviate significantly from a Poisson process [24], the times between request arrivals are given by a phase-type distribution with $a=16$ phases and a coefficient of variation close to 15 . The system capacity is $N=256$ requests (packets). The mean service time to process a packet is taken to be 1 .

Figure 4 illustrates the results obtained from our model. It shows the mean packet sojourn time (request response time) as a function of the mean rate of packet arrivals. For comparison, we have included the results obtained from our model in the case of a Poisson arrival stream. We observe, as could be expected, that the influence of the arrival process on the mean packet sojourn time is most visible at medium loads for which assuming Poisson arrivals would result in a significant underestimation of the mean packet sojourn time. Interestingly, for high load values the mean sojourn time with our phase-type distribution of time between arrivals can actually become lower than with Poisson arrivals. An examination of other performance metrics indicates that this is due to higher loss probabilities than with Poisson arrivals (and thus lower attained throughput, i.e., carried

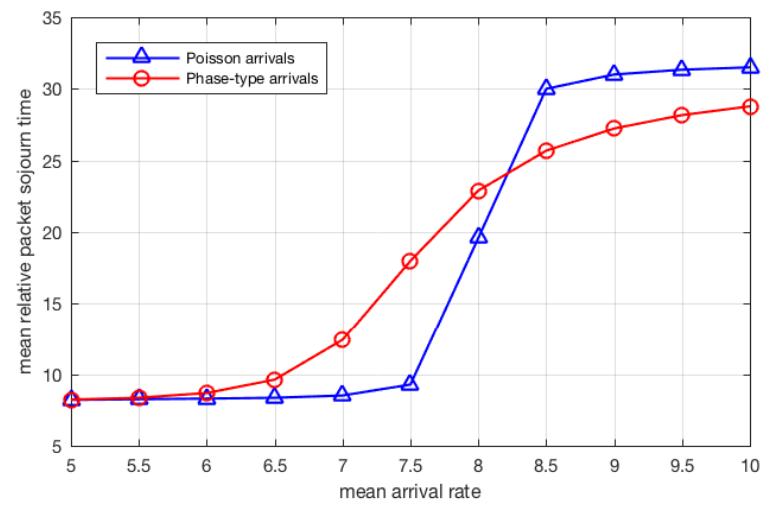

Fig. 4: Example II: mean relative packet sojourn time as a function of load for non-Poisson and Poisson arrival processes.

traffic). It is worthwhile noting that, with the threshold values used, there appears to be no visible "dips" in the mean sojourn time.

"Hard" threshold values used in this example are: $\gamma(9,1,0)=1, \gamma(19,2,0)=1, \gamma(29,3,0)=1, \gamma(39,4,0)=$ $1, \gamma(49,5,0)=1, \gamma(59,6,0)=1, \gamma(69,7,0)=1$, $\delta(6,2,0)=1, \delta(17,3,0)=1, \delta(27,4,0)=1, \delta(34,5,0)=1$, $\delta(41,6,0)=1, \delta(49,7,0)=1, \delta(55,8,0)=1$. These threshold values were selected to illustrate the possible influence of the arrival process on the packet sojourn time.

Figure 5 shows the inter-arrival time distribution used for this example.

\subsection{Example III: cloud-computing-like system}

In our third example, we consider a high-level model of a cloud-computing platform in which Virtual Machines (VMs) are added and removed dynamically in response to workload variations (e.g., auto-scaling feature in Amazon Elastic Compute Cloud (EC2)). Here, we assume that there is a sizeable delay when activating additional VMs, and we consider "hard" thresholds to activate (respectively, deactivate) VMs. We assume that request arrivals come from a set of $K=200$ exponential request sources resulting in a state-dependent arrival rate $\lambda(n)=(K-n) \phi$ where $\phi$ is the unitary request rate of an active source. The system capacity $N$ is greater than the number of request sources so that there are no lost requests. We assume that the number of VMs varies in the range 2 to $16(C=16)$ and VMs are added and removed in groups of 2, i.e., $c_{\ell}=2 \ell, \ell=1, \ldots, 8$. We fix the forward thresholds so as to switch to the next higher operating level when the relative request response time is around 7 and switch back to the preceding level when the relative response time is around 4 . Here, the mean relative response time is defined as the ratio $R / T$ where $T$ is the mean request execution time.

For our numerical study, we take $T=1$ and we explore the mean relative response time as a function of the unitary request rate $\phi$. Figure 6 a illustrates the results obtained for three values of the mean activation delay $1 / \omega=1,8$ and 16 . We note the presence of several "dips" and inflexion points corresponding to the VMs being activated and deactivated 

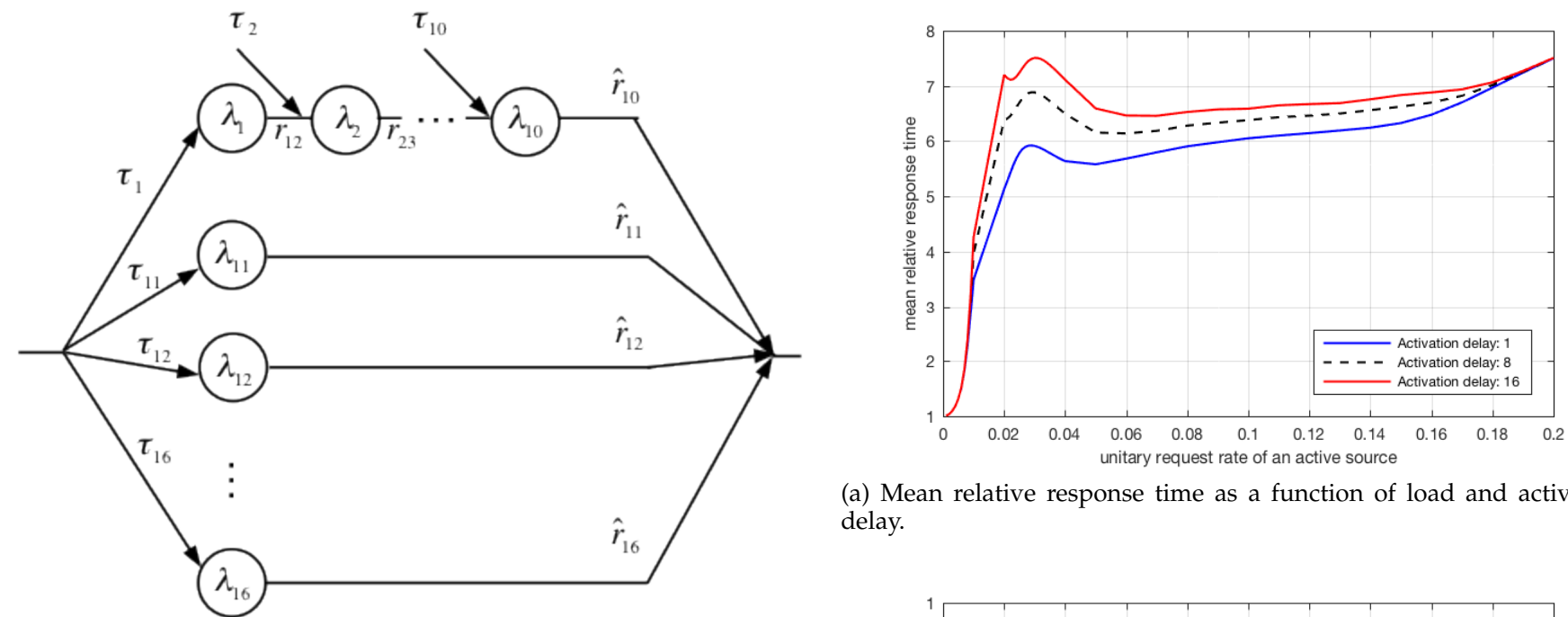

(a) Mean relative response time as a function of load and activation delay.

\begin{tabular}{|c|c|c|c|}
\hline \multicolumn{2}{|c|}{ Probabilities } & \multicolumn{2}{c|}{ Phase rate } \\
\hline$\tau_{1}$ & $4.96299789 \mathrm{e}-002$ & $\lambda_{1}$ & $7.02937615 \mathrm{e}+000$ \\
$\tau_{2}$ & $6.55622766 \mathrm{e}-002$ & $\lambda_{2}$ & $5.60291523 \mathrm{e}+000$ \\
$\tau_{3}$ & $6.08048526 \mathrm{e}-002$ & $\lambda_{3}$ & $5.01429942 \mathrm{e}+000$ \\
$\tau_{4}$ & $3.95306023 \mathrm{e}-002$ & $\lambda_{4}$ & $4.39854222 \mathrm{e}+000$ \\
$\tau_{5}$ & $8.43336270 \mathrm{e}-002$ & $\lambda_{5}$ & $4.11181239 \mathrm{e}+000$ \\
$\tau_{6}$ & $1.11445442 \mathrm{e}-001$ & $\lambda_{6}$ & $3.09159050 \mathrm{e}+000$ \\
$\tau_{7}$ & $4.34658002 \mathrm{e}-002$ & $\lambda_{7}$ & $2.59431915 \mathrm{e}+000$ \\
$\tau_{8}$ & $1.13779144 \mathrm{e}-002$ & $\lambda_{8}$ & $2.52635899 \mathrm{e}+000$ \\
$\tau_{9}$ & $3.87506920 \mathrm{e}-002$ & $\lambda_{9}$ & $2.45646593 \mathrm{e}+000$ \\
$\tau_{10}$ & $2.30172016 \mathrm{e}-001$ & $\lambda_{10}$ & $2.25907287 \mathrm{e}+000$ \\
$\tau_{11}$ & $5.27071506 \mathrm{e}-007$ & $\lambda_{11}$ & $2.23401704 \mathrm{e}-005$ \\
$\tau_{12}$ & $8.11915805 \mathrm{e}-006$ & $\lambda_{12}$ & $1.69154794 \mathrm{e}-004$ \\
$\tau_{13}$ & $1.08429620 \mathrm{e}-004$ & $\lambda_{13}$ & $9.82553880 \mathrm{e}-004$ \\
$\tau_{14}$ & $1.42476517 \mathrm{e}-003$ & $\lambda_{14}$ & $5.49823548 \mathrm{e}-003$ \\
$\tau_{15}$ & $1.86758284 \mathrm{e}-002$ & $\lambda_{15}$ & $3.05903719 \mathrm{e}-002$ \\
$\tau_{16}$ & $2.44709129 \mathrm{e}-001$ & $\lambda_{16}$ & $1.70040180 \mathrm{e}-001$ \\
$r_{12}, r_{23}, \ldots,=\hat{r}_{10}, \hat{r}_{11}, \ldots,=1$ \\
\hline \multicolumn{4}{|c|}{}
\end{tabular}

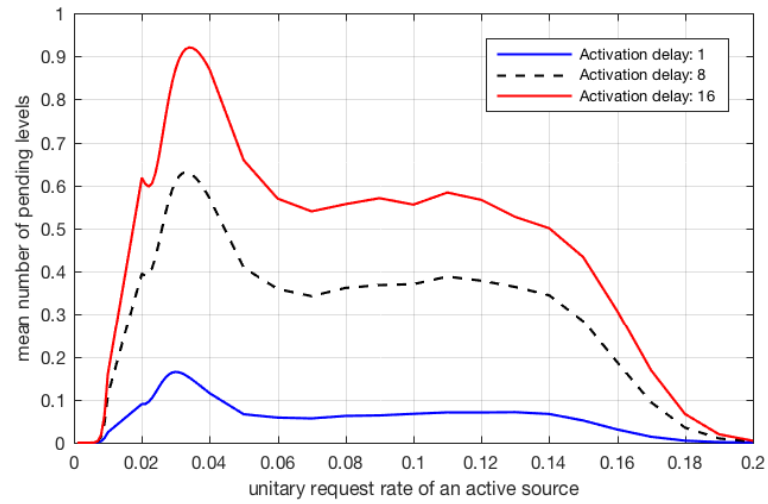

(b) Mean number of pending operating levels as a function of load and activation delay.

Fig. 6: Example III.

Fig. 5: Pareto-like distribution with $a=16$ phases for the time between arrivals in Example II.

in the cloud. We observe that, with the parameter values considered, the effect of the activation delay appears most visible near the value of the unitary request rate $\phi=0.03$. As shown in Figure 6b, it is near this value that the mean number of VMs whose activation is pending is the highest. For much lower loads, the system operates almost exclusively at level 1 and for much higher loads, the system operates almost exclusively at its highest level, so that the value of the activation delay matters little in these operating regions. While the mean response time is a recognized performance index, the distribution of the response time (in particular its tail) may also be an important consideration in the performance of a cloud-computing system. In Table 2 we show the results obtained from a set of discrete-event simulations of our model for low, medium and higher system loads. The results give the estimated percentages of response times not exceeding 0.25 times the mean, between 0.25 and 0.5 of the mean, etc, and finally exceeding 3 times the mean response time. For each value of model parameters, our simulation results are based on 700,000

request completions. For comparison, we have included the corresponding values for the exponential distribution with the same mean value. We observe, for the parameter values considered and under the assumptions of our model, that the response times only relatively rarely exceed twice the mean. We note that the exponential distribution seems a poor fit for the response times observed in our simulation.

We used the following "hard" threshold values in this example: $\gamma(14,1,0)=1, \gamma(28, \ell, 2-\ell)=1$ for $\ell=1,2$, $\gamma(42, \ell, 3-\ell)=1$ for $\ell=1,2,3, \gamma(56, \ell, 4-\ell)=1$ for $\ell=1, \ldots, 4, \gamma(70, \ell, 5-\ell)=1$ for $\ell=1, \ldots, 5, \gamma(84, \ell, 6-$ $\ell)=1$ for $\ell=1, \ldots, 6, \gamma(98, \ell, 7-\ell)=1$ for $\ell=1, \ldots, 7$, $\delta(12, \ell, 2-\ell)$ for $\ell=1,2, \delta(28, \ell, 3-\ell)$ for $\ell=1,2,3$, $\delta(32, \ell, 4-\ell)$ for $\ell=1, \ldots, 4, \delta(44, \ell, 5-\ell)$ for $\ell=1, \ldots, 5$, $\delta(58, \ell, 6-\ell)$ for $\ell=1, \ldots, 6, \delta(72, \ell, 7-\ell)$ for $\ell=1, \ldots, 7$, $\delta(86, \ell, 8-\ell)$ for $\ell=1, \ldots, 8$. These threshold values were chosen to illustrate the impact of server activation delays in the context of a system with multiple operating levels.

\subsection{Example IV: Linux-like system}

Our last example is devoted to a system inspired from a server running the Linux Operating System [25]. The server operates at $L=4$ levels corresponding to from 1 to 4 processor cores made available to execute requests $\left(c_{\ell}=\ell, \ell=1,2,3,4\right)$. We assume that the request arrival 
TABLE 2: Distribution of the response time in Example III with an activation delay $1 / \omega$ of 8 .

\begin{tabular}{ccccccccccc}
\hline Request rate & Mean $R$ & $\leq 0.25 R$ & $(0.25,0.5] R$ & $(0.5,0.75] R$ & $(0.75,1] R$ & $(1,1.5] R$ & $(1.5,2] R$ & $(2,2.5] R$ & $(2.5,3] R$ & $>3 R$ \\
\hline 0.01 & 3.9641 & $10.52 \%$ & $14.78 \%$ & $14.27 \%$ & $13.53 \%$ & $25.03 \%$ & $15.05 \%$ & $5.08 \%$ & $1.3 \%$ & $0.44 \%$ \\
corr. exp. dist. & same & $15.55 \%$ & $13.13 \%$ & $11.09 \%$ & $9.36 \%$ & $14.59 \%$ & $10.40 \%$ & $7.42 \%$ & $5.29 \%$ & $13.17 \%$ \\
\hline \hline 0.03 & 6.8843 & $0 \%$ & $0.26 \%$ & $8.44 \%$ & $44.95 \%$ & $44.04 \%$ & $2.27 \%$ & $0.03 \%$ & $0 \%$ & $0 \%$ \\
corr. exp. dist. & same & $25.36 \%$ & $18.93 \%$ & $14.13 \%$ & $10.55 \%$ & $13.75 \%$ & $7.66 \%$ & $4.27 \%$ & $2.38 \%$ & $2.99 \%$ \\
\hline \hline 0.15 & 6.6260 & $0 \%$ & $0.01 \%$ & $0.82 \%$ & $52.16 \%$ & $46.82 \%$ & $0.14 \%$ & $0.04 \%$ & $0 \%$ & $0 \%$ \\
corr. exp. dist. & same & $24.50 \%$ & $18.50 \%$ & $13.96 \%$ & $10.54 \%$ & $13.97 \%$ & $7.97 \%$ & $4.54 \%$ & $2.59 \%$ & $3.43 \%$ \\
\hline
\end{tabular}

process is non-Poisson with a coefficient of variation of the time between arrivals equal to 3 . We assume that the system capacity is limited to $N=128$ requests. Based on actual energy consumption measurements in a Dell Power Edge server (with Intel Xeon processors) [26], we assume that the energy consumption per time unit is given by a function of the form $b+c_{\ell} d$ where $b$ denotes the base power consumption when the system is idle, $c_{\ell}$ is the number of processors corresponding to level $\ell$ and $d$ is the average power consumption of a processor. We also assume that the activation time for adding processors is negligible. Here, we consider "hard" thresholds when activating and deactivating processor cores, i.e. switching operating levels.

Our goal is to determine threshold values that minimize a cost function combining system performance and energy consumption. Thus, our cost function comprises two components: energy cost and performance cost. For the former, since one processor is always active, we focus on the additional power consumption $\sum_{\ell=1}^{L} p(\ell)\left(c_{\ell}-1\right) d$. Taking this value relative to the maximum additional power consumption $\left(c_{L}-1\right) d$, we get as our energy cost $\eta_{e}=\frac{\sum_{\ell=1}^{L} p(\ell)\left(c_{\ell}-1\right)}{c_{L}-1}$. Note that this relative measure has a range of 0 to 1 . As a measure of performance cost, we use the relative request response time $H=R / T$ where $T=1 / \mu(1,1)$ is the mean execution time by a single processor core. We denote by $H_{1}$ and $H_{L}$ the corresponding relative response times in a single-level system with $c_{1}$ and $c_{L}$ processors, respectively. Clearly, we have $H_{L}<H<H_{1}$. We use $\eta_{p}=\frac{H-H_{L}}{H_{1}-H_{L}}$ as our performance cost measure. This relative measure, too, has a range of 0 to 1 . Our overall cost function is defined as a linear combination of these two components $\eta=\beta_{e} \eta_{e}+\beta_{p} \eta_{p}$. The coefficients $\beta_{p}$ and $\beta_{e}=1-\beta_{p}$ correspond to the relative importance we assign to performance versus energy consumption. Note that the first term in our cost function increases as the number of processors increases, while the opposite is true of the second term resulting in a tradeoff between performance and energy consumption.

In our numerical study, we take the mean execution time by a single processor core to be 1 and we consider two sets of values for the weighting factors in our cost function: $\left(\beta_{e}=0.85, \beta_{p}=0.15\right)$ and $\left(\beta_{e}=\beta_{p}=0.5\right)$. In exploring the values of thresholds, we concentrate on forward thresholds $(\gamma(n, \ell, k))$ and we fix backward thresholds $(\delta(n, \ell, k))$ at $4 / 5$ of the corresponding forward threshold. We consider three workload levels, referred to as low, medium and high. These workload levels correspond to mean arrival rates of $1.5,2.5$ and 3.5, respectively (recall that we assume a total of 4 processor cores). As illustrated in Figure 7a for $\beta_{p}=0.15$, the threshold values that minimize our cost function (determined under the constraint of a fixed relationship between forward and backward thresholds) depend on the workload

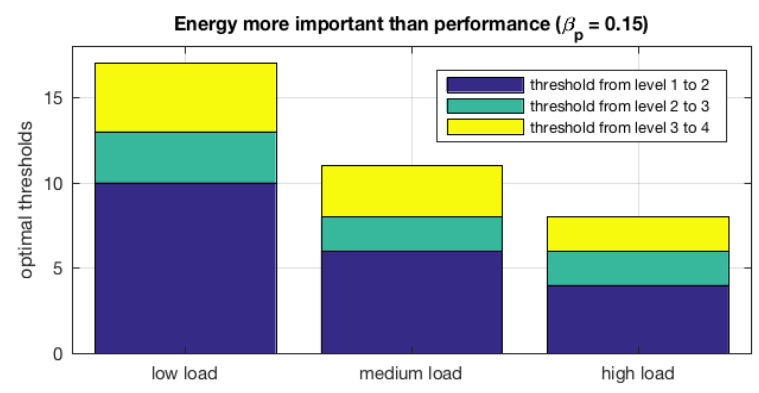

(a) Optimal forward thresholds for three load levels with $\beta_{p}=0.15$.

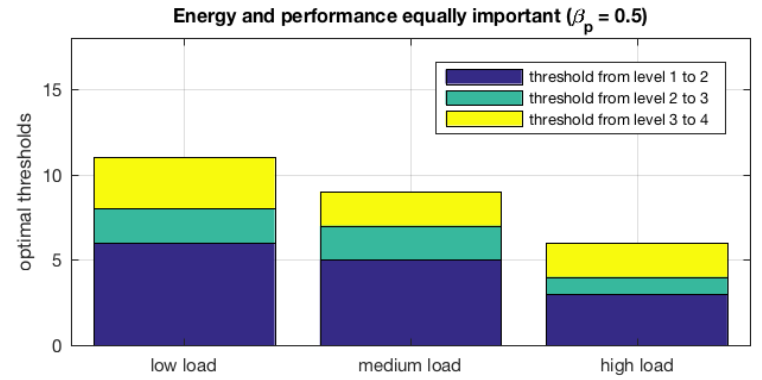

(b) Optimal forward thresholds for three load levels with $\beta_{p}=0.5$.

Fig. 7: Example IV: optimal thresholds.
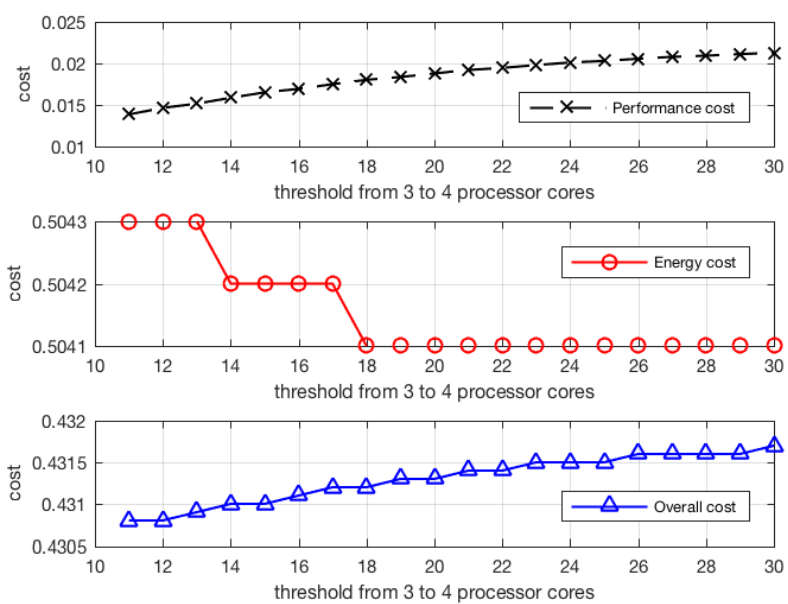

Fig. 8: Example IV: variation of cost components as a function of forward threshold between 3 and 4 processor cores. 
level. The higher the workload, the sooner a switch to higher operating level is required. Similar effect can be observed in Figure $7 \mathrm{~b}$ for $\beta_{p}=0.5$, and, as could be expected, with more emphasis on performance in this case, the threshold values tend to be smaller, i.e., switch to higher operating levels happens sooner. Finally, Figure 8 shows an example of how the components of our cost function vary as we increase the threshold value to switch from 3 to 4 cores, while keeping the other two threshold values constant. We observe that the relatively slow step-wise decrease in energy cost compared to the faster increase in our performance cost component. This figure was obtained with a mean request arrival rate of 2.5 and $\beta_{p}=0.15$.

Figure 9 shows the inter-arrival time distribution used for this example.

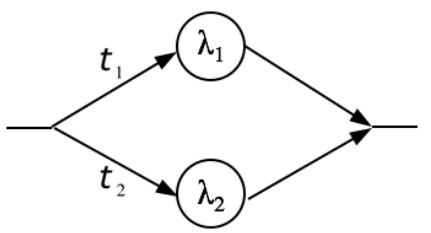

\begin{tabular}{|c|c|c|c|}
\hline \multicolumn{2}{|c|}{ Probabilities } & \multicolumn{2}{c|}{ Phase rate } \\
\hline$\tau_{1}$ & $2.8595933766 \mathrm{e}-005$ & $\lambda_{1}$ & $2.6666667 \mathrm{e}-003$ \\
$\tau_{2}$ & $1-\tau_{1}$ & $\lambda_{2}$ & 1.01081081 \\
\hline
\end{tabular}

Fig. 9: Hyperexponential distribution with $a=2$ phases for the time between arrivals in Example IV.

Note that in all our case studies, the stringency for the convergence of the fixed-point iterative solution of the model with Poisson or quasi-Poisson arrivals was set to $10^{-6}$ for the maximum relative difference in conditional probabilities. In the case of non-Poisson arrival, we used $\epsilon=10^{-4}$ (cf. Algorithm 1) for the relative difference between $\bar{n}$. Note also that the presence (or not) and location of "dips" and inflexion points depends on the values of thresholds at which switching between operating levels occurs.

\section{Conclusions}

We have presented a model of a system with multiple operating levels and possibly "soft" (probabilistic) thresholds for switching from one operating level to another. We assume an arbitrary number of servers and operating levels, each higher level corresponding to an arbitrary number of additional servers. We also assume a memoryless distribution for the request service times. Our model accounts for possible delays in level activation, hysteresis in level switching, as well as for general (phase-type) times between request arrivals. We propose a semi-numerical solution for our model where a set of conditional state probabilities is computed using a fixed-point iteration. Our solution approach uses simple mathematics. Although we do not have a proof of convergence of our fixed-point iteration, in the over 60,000 examples we studied the method never failed to converge within typically between a few tens to a few hundreds of iterations.

We use four case study examples, mostly inspired by features of real systems, to illustrate the flexibility of our model.
Our example inspired by the SMT feature in AIX accounts for the speed degradation of each server as the number of servers increases, as well as for arbitrary numbers of servers corresponding to different operating levels. Our example of Virtual Switches in the context of computer networks illustrates the potential importance of the arrival process on the performance of such systems. Our results indicate that the influence of the arrival process on the mean packet sojourn time is most visible at medium and high loads. Assuming Poisson arrivals would result in a significant underestimation of the mean packet sojourn time at medium loads, while at high loads it would underestimate packet losses. We use the example of Virtual Machine allocation in a cloud system to illustrate the potential importance of larger activation delays. Interestingly, the influence of activation delays is most visible for medium loads, which is most probably the desired operating region for these systems. Our example of processor core activation in a Linux-like environment clearly shows the dependence of optimal threshold values for switching between levels on system workload (in addition to the obvious dependence on the criterion used to determine such thresholds).

A natural extension of our work would be to attempt to relax the assumption of memoryless service times in our model.

\section{ACKNOWLEDGMENTS}

This work was supported in part by the LABEX MILYON (ANR-10-LABX-0070) of Université de Lyon, within the program "Investissements d'Avenir" (ANR-11-IDEX-0007) operated by the French National Research Agency (ANR). Part of this work was also supported by ANR MARMOTE (ANR-12-MONU-0019) and Télécom SudParis. The authors wish to thank Dr. Kenneth James for his helpful remarks and suggestions. The authors would like to express their sincere thanks to the anonymous referees for their remarks and comments.

\section{REFERENCES}

[1] A. Fox, R. Griffith, A. Joseph, R. Katz, A. Konwinski, G. Lee, D. Patterson, A. Rabkin, and I. Stoica, "Above the clouds: A berkeley view of cloud computing," Dept. Electrical Eng. and Comput. Sciences, University of California, Berkeley, Rep. UCB/EECS, vol. 28, no. 13, p. 2009, 2009.

[2] "VMWare ESX Server." http://www.vmware.com/products/esx. Accessed: 2017-04-20.

[3] "IBM AIX 7.2." http://www-03.ibm.com/systems/power/software/aix/. Accessed: 2017-04-20.

[4] H. Hawilo, A. Shami, M. Mirahmadi, and R. Asal, "Nfv: state of the art, challenges, and implementation in next generation mobile networks (vepc)," IEEE Network, vol. 28, no. 6, pp. 18-26, 2014.

[5] O. C. Ibe and J. Keilson, "Multi-server threshold queues with hysteresis," Performance Evaluation, vol. 21, no. 3, pp. 185-213, 1995.

[6] L. Golubchik and J. Lui, "Bounding of performance measures for a threshold-based queueing system with hysteresis," in ACM SIGMETRICS Performance Evaluation Review, vol. 25, pp. 147-157, ACM, 1997.

[7] J. C. Lui and L. Golubchik, "Stochastic complement analysis of multi-server threshold queues with hysteresis," Performance Evaluation, vol. 35, no. 1, pp. 19-48, 1999.

[8] L.-M. Le Ny and B. Tuffin, "A simple analysis of heterogeneous multi-server threshold queues with hysteresis," in Proceedings of the Applied Telecommunication Symposium, SCS, 2002. 
[9] I. Mitrani, "Service center trade-offs between customer impatience and power consumption," Performance Evaluation, vol. 68, no. 11, pp. 1222-1231, 2011.

[10] I. Mitrani, "Managing performance and power consumption in a server farm," Annals of Operations Research, pp. 1-14, 2013.

[11] F. Aitt-Salaht and H. Castel-Taleb, "The threshold based queueing system with hysteresis for performance analysis of clouds," in Computer, Information and Telecommunication Systems (CITS), 2015 International Conference on, pp. 1-5, IEEE, 2015.

[12] F. Aït-Salaht and H. Castel-Taleb, "Stochastic bounding models for performance analysis of clouds," in Computer and Information Technology; Ubiquitous Computing and Communications; Dependable, Autonomic and Secure Computing; Pervasive Intelligence and Computing (CIT/IUCC/DASC/PICOM), 2015 IEEE International Conference on, pp. 603-610, IEEE, 2015.

[13] A. O. Allen, Probablity, Statistics and Queueing Theory with Computer Science Applications, Second Edition. Elsevier, 1990.

[14] M. Harchol-Balter, Performance modeling and design of computer systems: queueing theory in action. Cambridge University Press, 2013.

[15] M. A. Johnson and M. R. Taaffe, The denseness of phase distributions. School of Industrial Engineering, Purdue University, 1988.

[16] A. Bobbio, A. Horváth, and M. Telek, "Matching three moments with minimal acyclic phase type distributions," Stochastic models, vol. 21, no. 2-3, pp. 303-326, 2005.

[17] T. Osogami and M. Harchol-Balter, "Closed form solutions for mapping general distributions to quasi-minimal ph distributions," Performance Evaluation, vol. 63, no. 6, pp. 524-552, 2006.

[18] A. Brandwajn, "Equivalence and decomposition in queueing systems—a unified approach," Performance Evaluation, vol. 5, no. 3, pp. 175-186, 1985.

[19] A. Brandwajn and T. Begin, "A recurrent solution of $P h / M / c / N$ like and $P h / M / c$-like queues," Journal of Applied Probability, vol. 49, pp. 84-99, March 2012.

[20] T. Atmaca, T. Begin, A. Brandwajn, and H. Castel-Taleb, "Performance evaluation of cloud computing centers with general arrivals and service," IEEE Transactions on Parallel and Distributed Systems, vol. 27, no. 8, pp. 2341-2348, 2016.

[21] A. Brandwajn and H. Wang, "A conditional probability approach to $M / G / 1$-like queues," Performance Evaluation, vol. 65 , no. 5, pp. $366-381,2008$

[22] "IBM AIX 7.2 Simultaneous multithreading." https://www.ibm.com/support/knowledgecenter/ssw aix 71/com.ibm.aix.genprogc/smt.htm. Accessed: 2017-04-20.

[23] N. Egi, G. Iannaccone, M. Manesh, L. Mathy, and S. Ratnasamy, "Improved parallelism and scheduling in multi-core software routers," The Journal of Supercomputing, pp. 1-29, 2013.

[24] V. Paxson and S. Floyd, "Wide area traffic: the failure of poisson modeling," IEEE/ACM Transactions on Networking (ToN), vol. 3, no. 3, pp. 226-244, 1995.

[25] "Debian GNU/Linux, kernel version 3.x."

[26] "Dell PowerEdge R815 Rack Server." http://www.dell.com/us/business/p/poweredge-r815/pd. Accessed: 2017-04-20.

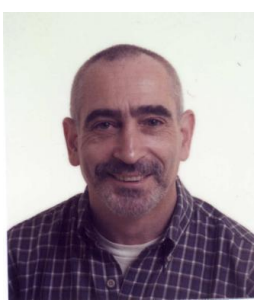

Alexandre Brandwajn holds a Ingénieur Civi des Télécommunications degree from the Ecole Nationale Supérieure des Télécommunications in Paris, and a Docteur d'Etat in Computer Science degree from the University of Paris VI. He worked as researcher at the Institut de Recherche en Informatique et Automatique (IRIA), France, then he was on the faculty of the Ecole Nationale Superieure des Telecommunications in Paris where he directed a project in adaptive computer architecture. Later he joined Amdahl Corporation in Sunnyvale, California, where he was a Senior Computer Architect, and then Manager of Systems Analysis group. Since 1985 he is a professor of Computer Engineering at the University of California at Santa Cruz. His current research interests include efficient solution of systems with large state space, application of conditional probability in the solution of performance models, models of virtualized systems, as well as efficient solution of priority systems.

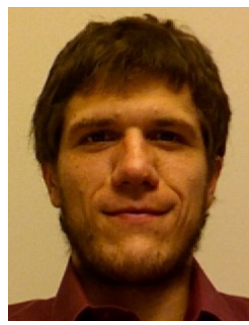

Thomas Begin received a M.Sc. degree in Electronics Engineering from ISEP (Paris) in 2003 and a M.Sc. and a Ph.D. degrees in Computer Science from UPMC (U. Paris 6) in 2005 and 2008 , respectively. He was a post-docotral fellow at UC Santa Cruz in 2009. Since 2009, he is Assistant Professor at UCBL (U. Lyon 1) in the Computer Science departement. During the academic year 2015-2016, he was on a research leave at the University of Ottawa (uOttawa). His research interests are in performance evaluation, computer network, and system modeling. His principal applications pertain to high-level modeling, wireless networks, resource allocation and queueing systems.

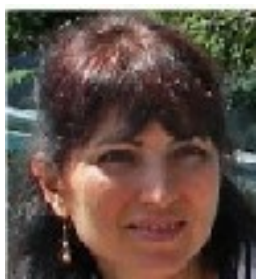

Hind Castel-Taleb is professor of computer networks at Telecom SudParis, and member of SAMOVAR laboratory. She obtained her habilitation in computer science at Paris VI (Université Pierre et Marie Curie, France) in 2011. Her main research interest is about performance evaluation of computer networks, traffic analysis, queueing theory, Markov chains analysis stochastic comparison methods.

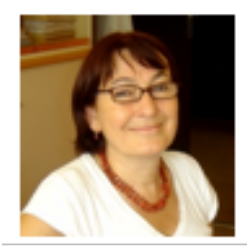

Tülin Atmaca received the Habilitation Degree in July 2008 from the University of Paris VI and the PhD degree in Computer Science from The University of Paris XI (ORSAY), in France in 1987. Since January 1992, she works at Institut Telecom/Telecom SudParis in France, and she is Full Professor. She was also Visiting Professor in the Computer Science Department of the North Carolina State University, as well as in the department of Industrial Engineering of the Rutgers University. She supervised more than $15 \mathrm{PhD}$ thesis, numerous Master thesis and Post-docs. Her research interests are the performance evaluation of telecommunication networks, the traffic and congestion control, and Quality of Services aspects in networks. Recently she also works on energy saving solutions in integrated access networks: optical and mobile networks. She has been involved in several national and international research projects in the field of the optical packet switching networks and their performance and also in industrial research projects with Alcatel Bell Labs and Orange, etc. She has conducted many research projects since several years with Central and Eastern Europe countries (Poland, Slovakia, Romania) and Turkey. She is also member of several scientific organisations and the author of 4 book chapters and more than 150 publications. She is also TPC member and co-chair of various international conferences. She is in Editorial Board of 3 journals (chief of one of them). 